\title{
Aspectos clínico-patológicos da estomatite urêmica: Uma revisão integrativa da
}

\section{literatura}

\author{
Clinical-pathological aspects of uremic stomatitis: An integrative literature review \\ Aspectos clínicos y patológicos de la estomatitis urémica: Revisión integradora de la literatura
}

Recebido: 01/04/2021 | Revisado: 10/04/2021 | Aceito: 15/04/2021 | Publicado: 28/04/2021

Matheus Andrade Rodrigues

ORCID: https://orcid.org/0000-0003-2501-6546 State University of Paraíba, Brasil

E-mail: matheusandrade1606@gmail.com

Ana Beatriz Barros dos Santos ORCID: https://orcid.org/0000-0002-5170-7554 State University of Paraíba, Brasil Email: abeatrizbs1@gmail.com

Fábio Gabriel de Sousa Carvalho ORCID: https://orcid.org/0000-0002-7777-4827 State University of Paraíba, Brasil

E-mail: fabio.carvalho@aluno.uepb.edu.br

Giselle Moreira de Carvalho

ORCID: https://orcid.org/0000-0003-4788-7254

State University of Paraíba, Brasil

E-mail: giselle.carvalho@aluno.uepb.edu.br

Lara Cristina de Albuquerque Carvalho

ORCID: https://orcid.org/0000-0002-6119-1811

State University of Paraíba, Brasil

E-mail: lara.carvalho@aluno.uepb.edu.br

Maria Ivaiane Boaventura de Sobral

ORCID: https://orcid.org/0000-0002-9092-528X

State University of Paraíba, Brasil

E-mail: ivaiane1011@gmail.com

Maria Izabela Brandão Vasconcelos

ORCID: https://orcid.org/0000-0002-2473-2789 State University of Paraíba, Brasil

E-mail: izabelabrandao18@gmail.com

Maria Vitória do Amaral Ferreira ORCID: https://orcid.org/0000-0003-3364-0365 State University of Paraíba, Brasil

E-mail: vitoriaamara1081@gmail.com

Matheus Harllen Gonçalves Veríssimo ORCID: https://orcid.org/0000-0003-2845-4832 State University of Paraíba, Brasil

E-mail: matheusharllen@gmail.com

Matheus Rodrigues dos Santos Arruda ORCID: https://orcid.org/0000-0002-6986-8225 State University of Paraíba, Brasil

E-mail: matheus.arruda@aluno.uepb.edu.br

Rayssa Ribeiro de Negreiros

ORCID: https://orcid.org/0000-0002-7870-971X State University of Paraíba, Brasil

E-mail: rayssa.negreiros@ aluno.uepb.edu.br

\section{Resumo}

Com o agravamento da insuficiência renal em um longo período de tempo, há o desenvolvimento de estomatite urêmica na cavidade bucal, sendo um processo patológico pouco estudo e reduzido número de pesquisas clínicas. Objetivo: Avaliar os aspectos clínico-patológicos e métodos de tratamento e remissão da estomatite urêmica através de uma revisão integrativa da literatura. Metodologia: Esta revisão integrativa da literatura possui uma metodologia qualitativa, sendo baseada no desenvolvimento da seguinte pergunta de pesquisa: Quais os principais aspectos clínico-patológicos da estomatite urêmica? Foram utilizadas três palavras-chave para a composição da chave de pesquisa, sendo os seguintes (MeSH/DeCS): ((Stomatitis uremic)) AND (Anatomy OR Pathology, Oral). Os critérios de elegibilidade foram os seguintes: artigos que se adequem à temática; pesquisa que envolvam aspectos anatômicos e/ou patológicos da estomatite urêmica; não houveram restrições quanto ao ano e idioma. Resultados: Dentre os aspectos clínicos, pode-se 
citar a presença de lesões esbranquiçadas no dorso e lateral da língua, assim como placas brancas distribuídas no assoalho da boca, que podem estar associadas ao hálito urêmico e xerostomia em alguns casos. Além dos aspectos clínicos, a estomatite urêmica apresenta alguns traços histológicos característicos: acantose com zona paraqueratótica espessa superficialmente, camada de queratinócitos dilatados profundamente, epitélio hiperplástico e hiperparaceratinização incomum. Assim, o diagnóstico pode ser realizado com base na observação dos aspectos clínicos e histológicos. O alívio e a remissão completa do processo patológico, frequentemente, são alcançados com o tratamento direcionado para a doença renal, como a hemodiálise, por exemplo. Conclusão: A presente revisão integrativa de literatura contou com a análise detalhada de 6 artigos e destacou os aspectos clínicos e patológicos da estomatite urêmica, incluindo os métodos de tratamento mais usados. Os resultados obtidos responderam com êxito a pergunta norteadora da pesquisa.

Palavras-chave: Estomatite; Anatomia; Patologia bucal.

\begin{abstract}
With the worsening of kidney failure over a long period of time, there is the development of uremic stomatitis in the oral cavity, a pathological process that has not been studied much and the number of clinical studies has been reduced. Objective: to evaluate the clinical-pathological aspects and methods of treatment and remission of uremic stomatitis through an integrative literature review. Methodology: This integrative literature review has a qualitative methodology, based on the development of the following research question: What are the main clinical and pathological aspects of uremic stomatitis? Three keywords were used to compose the research key, the following being (MeSH / DeCS): ((Uremic stomatitis)) AND (Anatomy OR Pathology, Oral). The eligibility criteria were as follows: articles that fit the theme; research involving anatomical and / or pathological aspects of uremic stomatitis; there were no restrictions on year and language. Results: Among the clinical aspects, we can mention the presence of whitish lesions on the back and side of the tongue, as well as white plaques distributed on the floor of the mouth, which may be associated with uremic breath and xerostomia in some cases. In addition to clinicians, an uremic stomatitis has some characteristic histological features: acanthosis with a superficially thick parakeratotic zone, a layer of deeply dilated keratinocytes, hyperplastic epithelium and unusual hyperparaceratinization. Thus, the diagnosis can be made based on the observation of clinical and histological aspects. Relief and complete remission of the pathological process are often achieved with targeted treatment for kidney disease, such as hemodialysis, for example. Conclusion: This integrative literature review included a detailed analysis of 6 articles and highlighted the clinical and pathological aspects of uremic stomatitis, including the most used treatment methods. The results obtained results successfully the guiding question of the research.
\end{abstract}

Keywords: Stomatitis; Anatomy; Pathology oral.

\title{
Resumen
}

Con el agravamiento de la insuficiencia renal durante un largo período de tiempo, se produce el desarrollo de estomatitis urémica en la cavidad bucal, proceso patológico poco estudiado y reducido número de estudios clínicos. Objetivo: evaluar los aspectos clínico-patológicos y métodos de tratamiento y remisión de la estomatitis urémica a través de una revisión integradora de la literatura. Metodología: Esta revisión integradora de la literatura tiene una metodología cualitativa, basada en el desarrollo de la siguiente pregunta de investigación: ¿Cuáles son los principales aspectos clínicos y patológicos de la estomatitis urémica? Se utilizaron tres palabras clave para componer la clave de investigación, siendo las siguientes (MeSH / DeCS): ((Estomatitis urémica)) Y (Anatomía O Patología, Oral). Los criterios de elegibilidad fueron los siguientes: artículos que se ajustan al tema; investigación que involucre aspectos anatómicos y / o patológicos de la estomatitis urémica; no hubo restricciones de año e idioma. Resultados: Entre los aspectos clínicos, podemos mencionar la presencia de lesiones blanquecinas en el dorso y lateral de la lengua, así como placas blancas distribuidas en el piso de la boca, que pueden estar asociadas a aliento urémico y xerostomía en algunos casos. casos. Además de los médicos, una estomatitis urémica tiene algunos rasgos histológicos característicos: acantosis con una zona paraqueratósica superficialmente gruesa, una capa de queratinocitos profundamente dilatados, epitelio hiperplásico e hiperparaceratinización inusual. Así, el diagnóstico se puede realizar a partir de la observación de aspectos clínicos e histológicos. El alivio y la remisión completa del proceso patológico a menudo se logran con un tratamiento específico para la enfermedad renal, como la hemodiálisis, por ejemplo. Conclusión: Esta revisión integradora de la literatura incluyó un análisis detallado de 6 artículos y destacó los aspectos clínicos y patológicos de la estomatitis urémica, incluidos los métodos de tratamiento más utilizados. Los resultados obtenidos dan como resultado con éxito la pregunta orientadora de la investigación.

Palabras clave: Estomatitis; Anatomía; Patología bucal.

\section{Introdução}

A estomatite urêmica consiste em um processo patológico manifestado na mucosa oral, apresentada pela primeira vez por Lanceraux (1887), e pouco descrita na literatura científica até os dias atuais. Segundo Cardoso et al. (2020) encontra-se intimamente relacionada com a insuficiência renal de longa data e agressivas alterações urêmicas no organismo. Ademais, Barie 
(1889), em seus estudos, descreveu quatro formas predominantes de estomatite urêmica: erite-hemopultácea, ulcerativa, hemorrágica e hiperceratótica.

Os aspectos anatômicos da estomatite são apresentados de formas diferentes nos pacientes. Uma característica em comum na maioria dos pacientes avaliados nessa revisão, são lesões esbranquiçadas na cavidade oral, como mostra McCreary et al. (1997). Conforme Larato (1975), alguns pacientes com estomatite urêmica, apresentaram lábios ressecados e inchados, com presença de úlceras e sangramento nas comissuras labiais. Foi observado também úlceras na mucosa jugal e nas laterais da língua.

De acordo com Jaspers (1975), a uremia, uma síndrome clínica na qual ocorre o acúmulo de escórias metabólicas nitrogenadas no sangue, que pode ser causada por insuficiência renal aguda ou crônica, está intimamente associada à estomatite urêmica. Entretanto, devido ao advento das técnicas artificiais de filtragem sanguínea como, por exemplo, a diálise renal, os estudiosos Depner (2001) e McCreary et al. (1997) consideram a estomatite urêmica como um achado clínico atípico.

Isto posto, o presente estudo teve como objetivo executar uma revisão integrativa de literatura no que diz respeito à estomatite urêmica, ressaltando seus aspectos clínico-patológicos, bem como os métodos efetivos para o tratamento e remissão da mesma.

\section{Metodologia}

Esta revisão integrativa da literatura possui uma metodologia qualitativa, sendo baseada em Rohter (2007), Souza, Silva e Carvalho (2010) e Pereira et al. (2018), e no desenvolvimento da seguinte pergunta de pesquisa: Quais os principais aspectos clínico-patológicos da estomatite urêmica?

Para isto, foi utilizada uma base de dados eletrônica: PubMed (U. S. National Library of Medicine (NLM)) e ScienceDirect (Science, Health and Medical Journals) para pesquisar e identificar estudos que respondessem à pergunta norteadora desta revisão integrativa da literatura.

Esta revisão integrativa baseou-se em cinco etapas: Na primeira etapa foi o estabelecimento dos descritores para ambas as bases de dados, sendo uma com a utilização de MeSH terms (PubMed) e com DeCS (ScienceDirect). Em seguida, segunda etapa, fora feito a busca avançada nas bases e análise do quantitativo dos artigos científicos presentes na íntegra. Logo em seguida, na terceira etapa, foram selecionados os artigos que se adequaram aos critérios de elegibilidade estabelecidos pelos pesquisadores. Na quarta e quinta etapa, os pesquisadores formularam uma tabela descritiva sobre os autores, objetivo da pesquisa, protocolo, resultados e conclusão e em seguida, desenvolvimento da discussão dos artigos científicos para uma análise do melhor manejo do clareamento dental, a fim de se chegar ao sucesso clínico e responder à pergunta norteadora estabelecida no início desta metodologia.

Foram utilizadas três palavras-chave para a composição da chave de pesquisa, sendo os seguintes (MeSH/DeCS): ((Stomatitis uremic)) AND (Anatomy OR Pathology, Oral). Em seguida, os pesquisadores selecionaram os trabalhos com análise no título e resumo, com base nos critérios de elegibilidade. Os critérios de elegibilidade foram os seguintes: artigos que se adequem à temática; pesquisa que envolvam aspectos anatômicos e/ou patológicos da estomatite urêmica; não houveram restrições quanto ao ano e idioma.

Também foi utilizado o sistema de formulário avançado para busca e seleção dos artigos utilizando conector booleano "AND”. Em seguida, artigos que preencheram os critérios de elegibilidade foram identificados e incluídos na revisão. Os trabalhos que preencheram todos os critérios de seleção foram incluídos no estudo, os que não preencheram os critérios e/ou não se mostraram relevantes foram excluídos. 


\section{Resultados e Discussão}

A estomatite urêmica é uma manifestação bucal do processo patológico agravado pela disfunção renal. Sendo assim, na maioria dos casos, é através do tratamento da doença renal crônica que haverá a redução ou total remissão dessa manifestação bucal (Talish \& DiLorenzo, 2020). Dessa forma, é necessária a análise das principais características clínicas e patológicas sobre a estomatite urêmica e os principais métodos de tratamento. Com a busca avançada através da utilizados dos descritores (MeSH/DeCS) já mencionados junto com o conector booleano, foram selecionados apenas os artigos que entraram nos critérios de inclusão. Os resultados por análise foram representados na Tabela 1:

Tabela 1 - Seleção dos artigos por análise empregada e estabelecimento dos critérios de inclusão.

\begin{tabular}{ccccc} 
& Íntegra & $\begin{array}{c}\text { Duplicado } \\
\text { s }\end{array}$ & $\begin{array}{c}\text { Adequados } \\
\text { aos critérios }\end{array}$ & Artigos selecionados \\
\hline PubMed & 9 & 0 & 8 & 4 \\
\hline $\begin{array}{c}\text { ScienceDire } \\
\text { ct }\end{array}$ & 397 & 3 & 14 & 2 \\
\hline
\end{tabular}

Fonte: Autores (2020).

Dos 22 artigo que se adequaram aos critérios, apenas 6 foram selecionados para compor o estudo, já que 16 artigos não retratavam diretamente os aspectos clínicos e patológicos, assim como o tratamento da Estomatite urêmica. Para isso, estabeleceu-se a construção da Tabela 2 aos estudos clínicos selecionados, com formulação das colunas (Autor/Ano; Objetivo do estudo; Protocolo; Resultados; Conclusão).

Tabela 2 - Relatos de casos detalhados em tabela de resultados.

\begin{tabular}{|c|c|c|c|c|}
\hline Autor/Ano & Caso clínico & Aspectos clínicos & Aspectos patológicos & Tratamento \\
\hline $\begin{array}{l}\text { McCreary } \\
\text { et al. } 1997 .\end{array}$ & $\begin{array}{l}\text { Paciente do sexo } \\
\text { masculino com } 48 \\
\text { anos, que chegou } \\
\text { ao ambulatório } \\
\text { com suspeita de } \\
\text { leucoplasia pilosa, } \\
\text { quando, na } \\
\text { verdade, tratou-se } \\
\text { de um caso de } \\
\text { estomatite urêmica. }\end{array}$ & $\begin{array}{l}\text { Inúmeras lesões } \\
\text { esbranquiçadas na cavidade } \\
\text { oral, envolvendo o dorso e a } \\
\text { lateral da língua, com } \\
\text { comprometimento } \\
\text { considerável da mucosa } \\
\text { oral. Sem a presença de } \\
\text { hálito urêmico. }\end{array}$ & $\begin{array}{l}\text { Acantose com uma zona } \\
\text { paraqueratótica espessa na } \\
\text { superfície e uma camada de } \\
\text { queratinócitos dilatados } \\
\text { profundamente a este. }\end{array}$ & $\begin{array}{l}\text { A realização da hemodiálise } \\
\text { minimizou as lesões orais a partir da } \\
\text { primeira semana, e as extinguiu após } \\
\text { um período de } 8 \text { semanas. }\end{array}$ \\
\hline $\begin{array}{l}\text { Leão, et al. } \\
\text { (2005) }\end{array}$ & $\begin{array}{l}\text { Paciente do sexo } \\
\text { masculino, } 46 \\
\text { anos, com queixa } \\
\text { de ardência na } \\
\text { mucosa oral e } \\
\text { disgeusia. }\end{array}$ & $\begin{array}{l}\text { O exame extra-oral não } \\
\text { revelou sinais óbvios de } \\
\text { doença renal, além de um } \\
\text { mau hálito urêmico. Não } \\
\text { havia linfadenopatia } \\
\text { cervical e os nervos } \\
\text { cranianos estavam } \\
\text { totalmente intactos. O } \\
\text { exame intra-oral revelou } \\
\text { placas brancas aderentes no } \\
\text { assoalho da boca, mucosas } \\
\text { bucais, bordas laterais da } \\
\text { língua e gengivas. }\end{array}$ & $\begin{array}{l}\text { O exame histopatógico, } \\
\text { através de biópsias } \\
\text { incisionais das bordas } \\
\text { esquerda e direita da língua, } \\
\text { revelou um epitélio } \\
\text { marcadamente acantótico, } \\
\text { com a maioria das camadas } \\
\text { suprabasais compreendendo } \\
\text { queratinócitos degenerados } \\
\text { de coloração pálida. As } \\
\text { camadas superficiais } \\
\text { apresentavam } \\
\text { descamação em alguns } \\
\text { pontos e havia hiperplasia do } \\
\text { componente basocelular. }\end{array}$ & $\begin{array}{l}\text { Como a doença renal estava } \\
\text { geralmente estável, não houve } \\
\text { necessidade de modificar o tratamento } \\
\text { sistêmico relevante, e um } \\
\text { enxaguatório bucal com peróxido de } \\
\text { hidrogênio a } 10 \% \text { (1: } 1 \text { em água) } 4 \\
\text { vezes ao dia foi prescrito como terapia } \\
\text { paliativa. após } 2 \text { semanas, as lesões } \\
\text { brancas desapare-ceram quase total- } \\
\text { mente com redução acentuada da } \\
\text { sensação de queimação e alteração do } \\
\text { paladar. }\end{array}$ \\
\hline
\end{tabular}




\begin{tabular}{|c|c|c|c|c|}
\hline $\begin{array}{l}\text { Antoniades, } \\
\text { Andreadis e } \\
\text { Grekas, } \\
2006 .\end{array}$ & 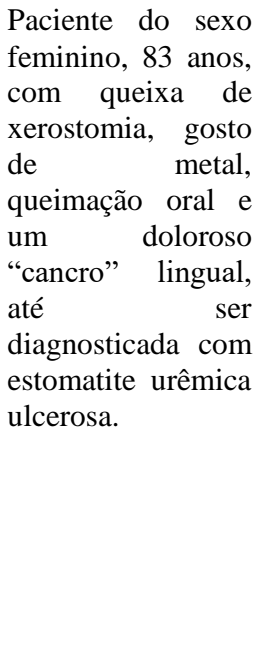 & $\begin{array}{l}\text { No exame intraoral, foi } \\
\text { observado uma extensa } \\
\text { lesão ulcerativa na } \\
\text { superfície dorsal anterior da } \\
\text { língua, coberta por espessa } \\
\text { camada amarela de crosta. } \\
\text { A mucosa da superfície } \\
\text { ventral da língua era } \\
\text { homogênea e branca e o } \\
\text { restante lingual } \\
\text { avermelhada e atrófica. } \\
\text { Existiam numerosos } \\
\text { capilares dilatados na } \\
\text { superfície ventral e no } \\
\text { assoalho da boca, além de } \\
\text { finas linhas esbranquiçadas } \\
\text { presentes na mucosa } \\
\text { esquerda. }\end{array}$ & $\begin{array}{l}\text { Os achados histopatoló- } \\
\text { gicos demonstraram uma } \\
\text { grande área de necrose } \\
\text { epitelial, coberta por uma } \\
\text { fina crosta pseudomem- } \\
\text { branosa. }\end{array}$ & $\begin{array}{l}\text { Após internação, foi iniciado um } \\
\text { tratamento conservador com } \\
\text { hidratação aumentada em progressão } \\
\text { de ringer isotônico e soluções de } \\
\text { lactulose, diuréticos e eletrólitos. A } \\
\text { paciente também recebeu tratamento } \\
\text { específico com miconazol em gel, } \\
\text { peróxido de hidrogênio e complexo de } \\
\text { vitamina B para suas lesões orais. } \\
\text { Dentro de } 10 \text { dias o tratamento } \\
\text { combinado com a higiene oral levou à } \\
\text { remissão absoluta da vermelhidão e a } \\
\text { ausência das lesões orais em } 15 \text { dias no } \\
\text { máximo. }\end{array}$ \\
\hline $\begin{array}{l}\text { Liao, Wu e } \\
\text { Chu (2017) }\end{array}$ & $\begin{array}{l}\text { Paciente do sexo } \\
\text { feminino, } 42 \text { anos, } \\
\text { com história de } \\
\text { nefropatia por IgA } \\
\text { com doença renal } \\
\text { crônica, estágio } 5 \text { e } \\
\text { hipertensão } \\
\text { apresentada com } \\
\text { pouco apetite, } \\
\text { coceira generali- } \\
\text { zada e disgeusia } \\
\text { com diminuindo a } \\
\text { sensação ao sal. }\end{array}$ & $\begin{array}{l}\text { O exame intraoral revelou a } \\
\text { presença de placas brancas } \\
\text { predominantemente } \\
\text { envolvendo as bordas } \\
\text { laterais e superfície dorsal } \\
\text { da língua, assoalho da boca } \\
\text { e mucosas bucais. }\end{array}$ & $\begin{array}{l}\text { O laudo patológico da lesão } \\
\text { não estava disponível } \\
\text { porque o paciente recusou a } \\
\text { biópsia. }\end{array}$ & $\begin{array}{l}\text { O tratamento consistiu em } \\
\text { hemodiálise e aumento da higiene oral } \\
\text { com anti-séptico enxaguatórios bucais } \\
\text { e agentes antimicrobianos / } \\
\text { antifúngicos se necessário. }\end{array}$ \\
\hline $\begin{array}{l}\text { Yano } \\
\text { Kinjo } \\
(2019)\end{array}$ & $\begin{array}{lr}\text { Paciente do } & \text { sexo } \\
\text { masculino, } & 37 \\
\text { anos, apresentou } \\
\text { fadiga, disgeusia e } \\
\text { anorexia por } 2 \\
\text { meses. } \\
\text { trabalhava como } \\
\text { encanador } \\
\text { fumava cinco } \\
\text { cigarros por dia } \\
\text { durante } 17 \text { anos. }\end{array}$ & $\begin{array}{l}\text { Placa branca na borda da } \\
\text { língua era notável. }\end{array}$ & $\begin{array}{l}\text { Epitélio hiperplásico e } \\
\text { incomum } \\
\text { hiperparaceratinização. }\end{array}$ & $\begin{array}{l}\text { A hemodiálise foi iniciada junto com } \\
\text { alfacalcidol, carbonato de lantânio e } \\
\text { darbepoetina alfa. As manchas na } \\
\text { língua desapareceram após } 1 \text { semana } \\
\text { depois terapia de substituição renal. }\end{array}$ \\
\hline $\begin{array}{l}\text { Talish e } \\
\text { DiLorenzo } \\
(2020)\end{array}$ & $\begin{array}{l}\text { Paciente do sexo } \\
\text { masculino, } 26 \text { anos } \\
\text { apresentou-se ao } \\
\text { pronto-socorro } \\
\text { com uma história } \\
\text { de } 2 \text { meses de uma } \\
\text { sensação de } \\
\text { paladar alterada e } \\
\text { descamação } \\
\text { indolor da língua } \\
\text { associada a mal- } \\
\text { estar, perda de peso } \\
\text { e músculo cólicas. } \\
\text { Ele tinha história } \\
\text { de doença renal } \\
\text { crônica atribuída a } \\
\text { refluxo vesico } \\
\text { ureteral. }\end{array}$ & $\begin{array}{l}\text { O exame físico mostrou } \\
\text { placas brancas, bem } \\
\text { demarcadas, aderentes nas } \\
\text { laterais e sob a superfície da } \\
\text { língua. }\end{array}$ & $\begin{array}{l}\text { O laudo patológico não foi } \\
\text { realizado. }\end{array}$ & $\begin{array}{l}\text { A resolução das lesões da língua foi } \\
\text { observada após três sessões de } \\
\text { hemodiálise. }\end{array}$ \\
\hline
\end{tabular}


No relato de McCreary et al. (1997), os autores analisaram as manifestações orais da estomatite urêmica de um paciente masculino de 48 anos de idade com suposto diagnóstico de Leucoplasia Pilosa. A confirmação do diagnóstico definitivo de estomatite urêmica mostrou-se possível através da percepção dos aspectos clínicos, que incluem: lesões esbranquiçadas na cavidade oral com comprometimento do dorso e lateral da língua, além da mucosa oral; e dos aspectos histológicos, sendo estes: acantose com zona paraqueratótica espessa superficialmente, além de uma camada de queratinócitos dilatados profundamente. Desse modo, o tratamento consistiu na realização da hemodiálise, minimizando as lesões orais a partir da primeira semana, e as extinguindo após um período de 8 semanas.

Leão et al. (2005) relataram o caso de um paciente de 46 anos que apresentava queixas de ardência em sua mucosa oral e disgeusia. Os exames realizados contêm traços que comprovam a estomatite urêmica, foram percebidos aspectos como mau hálito urêmico e placas brancas no assoalho da boca, nas bordas laterais da língua e gengivas. Mediante biópsias incisionais nas bordas esquerda e direita da língua percebeu-se um epitélio acantótico com a maioria das camadas suprabasais compreendendo queratinócitos degenerados e de coloração marcadamente pálida, somado a isso, nas camadas superficiais constavam descamações em alguns pontos e hiperplasia do componente basocelular. Tendo em vista as análises realizadas, o tratamento efetuado consistiu em condicionar o paciente ao enxaguatório bucal com peróxido de hidrogênio a 10\% (1:1 em água) por 4 vezes ao dia como terapia paliativa. Logo, após duas semanas constatou-se o desaparecimento das lesões quase que totalmente e redução acentuada da sensação de queimação e alteração do paladar.

No relato de Demetrios et al. (2006), os pesquisadores descrevem o caso de uma mulher de 83 anos encaminhada à clínica apresentando queixa de xerostomia, gosto de metal, sensação de queimação na boca e a presença, há aproximadamente três meses, de um "cranco" doloroso na região da língua. A partir do exame intraoral, foi-se identificada a presença, na região dorsal anterior da língua, de uma lesão ulcerativa extensa, coberta por uma camada de crosta amarelada. A mucosa da superfície ventral era homogênea e branca, com o restante lingual apresentando atrofia e coloração avermelhada.

Além disso, havia a presença de numerosos capilares dilatados na superfície ventral e no assoalho da boca, bem como linhas esbranquiçadas e finas, presentes no lado esquerdo da mucosa. Quando realizados exames histopatológicos, constatou-se a presença de uma região epitelial em estado de necrose sendo recoberta por uma crosta pseudomembranosa. Confirmado o diagnóstico de estomatite urêmica ulcerosa, e em razão de distúrbios pré-existentes, a paciente foi internada para a realização de um tratamento conservador que contava com hidratação aumentada; quanto às lesões orais, essas foram tratadas com miconazol em gel, peróxido de hidrogênio e complexo de vitamina B. O tratamento, combinado com a higiene oral, acarretou na ausência da vermelhidão dentro dos 10 primeiros dias e na remissão absoluta das lesões orais no período de 15 dias (Demetrios et al., 2006).

No que concerne aos estudos de Liao, Wu e Chu (2017), observou-se um estágio avançado da Doença Renal Crônica (DRC) em grau 5 agravada por um histórico de nefropatia por IgA e disgeusia. Quanto aos aspectos clínicos relatados, é notável a presença das particularidades já citadas por outros autores como a presença de placas brancas, predominantemente, envolvendo as bordas laterais e superfície dorsal da língua, assoalho da boca e mucosas bucais. Dessa maneira, os procedimentos realizados para tratar o processo patológico foram: hemodiálise, como forma de amenizar o agravamento causado pela DRC nos rins, antisséptico, enxaguatórios bucais e agentes antimicrobianos/antifúngicos para contribuir na diminuição das lesões bucais apresentadas.

Yano e Kinjo (2019) relataram um paciente do sexo masculino de 37 anos, fumante há 17 anos, que apresentava fadiga, disguesia e anorexia há 2 meses. As manifestações bucais apresentadas incluíam placas brancas visíveis nas bordas da língua e hálito de amônia, e nos aspectos histológicos estavam presentes epitélio hiperplástico e hiperparaceratinização incomum. A Tomografia Computadorizada mostrou rins atróficos bilaterais, indicando insuficiência renal em estágio final de etiologia 
desconhecida. A hemodiálise foi iniciada juntamente com alfacalcidol, carbonato de lantânio e darbepoetina alfa. Seu apetite e paladar voltaram ao normal, e as manchas brancas em sua língua desapareceram uma semana após a terapia de substituição renal.

No relato de caso de Talish e DiLorenzo (2020), os autores apresentaram um paciente de 26 anos com o nível de creatina de $22 \mathrm{mg}$ por decilitro e o nível de nitrogênio da ureia no sangue de mais de $225 \mathrm{mg}$ por decilitro. Com esses dados, seu histórico de doença renal crônica e as características observadas no exame físico, as quais eram: placas brancas, bem demarcadas e aderentes nas laterais e na face inferior da língua, foi confirmada a estomatite urêmica, levando a iniciação da hemodiálise. Com este procedimento, obteve-se como resultado a resolução das lesões da língua, observadas após três sessões. A partir disso, o paciente continuou em hemodiálise e em avaliação para o transplante renal.

Por se tratar de uma complicação intrabucal, o Cirurgião-Dentista assume papel fundamental na identificação dos traços patológicos e no tratamento da doença, tendo em vista que as manifestações apresentadas são comumente lesões esbranquiçadas no dorso e lateral da língua, assim como placas brancas distribuídas no assoalho da boca, que podem estar associadas ao hálito urêmico e xerostomia em alguns casos.

Além dos aspectos clínicos a estomatite urêmica apresenta alguns traços histológicos característicos: acantose com zona paraqueratótica espessa superficialmente, camada de queratinócitos dilatados profundamente, epitélio hiperplástico e hiperparaceratinização incomum. Assim, o diagnóstico pode ser realizado com base na observação dos aspectos clínicos e histológicos. Logo, a presente revisão integrativa de literatura contou com a análise detalhada de 6 artigos e destacou os aspectos clínicos e patológicos da estomatite urêmica, incluindo os métodos de tratamento mais usados. Os resultados obtidos responderam com êxito a pergunta norteadora da pesquisa.

\section{Considerações Finais}

A estomatite urêmica é considerada um processo patológico relativamente atípico e resultado das complicações associadas à insuficiência renal. Os trabalhos encontrados na literatura descrevem quatro formas principais da doença: estomatite hemorrágica, ulcerativa, erite-hemopultácea e hiperceratótica.

O alívio e a remissão completa do processo patológico, frequentemente, são alcançados com o tratamento direcionado para a doença renal, como a hemodiálise, por exemplo. Além das medidas com o objetivo de regular as funções renais, pode haver a associação de cuidados paliativos como a higiene bucal aumentada com a utilização de peróxido de hidrogênio, o uso de miconazol em gel e a ingestão de complexo de vitamina B para as lesões orais.

\section{Referências}

Antoniades, D., Markopoulos, A. K., \& Andreadis, D. et al. (2006). Ulcerative uremic stomatitis associated with untreated chronic renal failure: Report of a case and review of the literature. Oral Surgery, Oral Medicine, Oral Pathology, Oral Radiology, and Endodontology, 101(5): 608-13.

Barie, E. (1889). De la stomatite uremique. Archives of General Internal Medicine, 2:415-32.

Depner, T. A. (2001). Uremic toxicity: urea and beyond. Sem Dial, 14:246-51.

Cardoso, F. B., et al. (2020). Estomatite urémica em um paciente pós-transplante de fígado: um relato de caso. Cirurgia Oral, Medicina Oral, Patologia Oral e Radiologia Oral, 129(1): e125.

Jaspers, M. T. (1975). Unusual oral lesions in an uremic patient. Review of the literature and report of a case. Oral Surgery, Oral Medicine, Oral Pathology, and Oral Radiology, 39:934-44.

Lancereaux, M. (1887). Anatomie pathologique et complications de 1' uremia. Union Med, 44:829-30.

Larato, D. C. (1975). Uremic stomatitis: report of a case. Journal of Periodontology, 46(12): 731-3.

Leão, J. C., et al. (2005). Uremic stomatitis in chronic renal failure. Clinics, 60(3): 259-262.

Liao, C. Y., Wu, C. C., \& Chu, P. L. (2017). Uremic stomatitis. QJM: An International Journal of Medicine, 110(4): $247-248$. 
Research, Society and Development, v. 10, n. 5, e8510514547, 2021

(CC BY 4.0) | ISSN 2525-3409 | DOI: http://dx.doi.org/10.33448/rsd-v10i5.14547

McCreary, C. E., et al. (1997). Uremic stomatitis mimicking oral hairy leukoplakia: report of a case. Oral Surgery, Oral Medicine, Oral Pathology, and Oral Radiology, 83:350-3.

Talish, M., \& DiLorenzo, A. M. (2020). Uremic Stomatitis. The New England journal of medicine, 382(26), 2556.

Yano, H., \& Kinjo, M. (2019). Uraemic stomatitis. BMJ Case Rep, 12(10): e231948. 